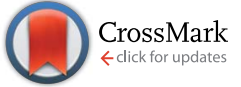

Cite this: Chem. Sci., 2015, 6, 6986

Received 9th July 2015

Accepted 26th August 2015

DOI: $10.1039 / \mathrm{c} 5 \mathrm{sc} 02485 \mathrm{~d}$

www.rsc.org/chemicalscience

\section{Chiral ion-pair organocatalyst promotes highly enantioselective 3-exo iodo-cycloetherification of allyl alcohols $\uparrow$}

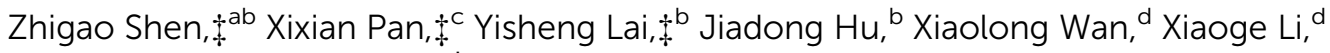 \\ Hui Zhang ${ }^{\mathrm{e}}$ and Weiqing Xie Ka $^{\text {Z }}$
}

By designing a novel chiral ion-pair organocatalyst composed of chiral phosphate and DABCO-derived quaternary ammonium, highly enantioselective 3-exo iodo-cycloetherification of allyl alcohols was achieved using NIS as a halogen source. Based on this reaction, one-pot asymmetric 3-exo iodocycloetherification/Wagner-Meerwein rearrangement of allyl alcohols en route to enantioenriched 2-iodomethyl-2-aryl cycloalkanones was subsequently developed. Due to the participation of adjacent iodine, the Wagner-Meerwein rearrangement of 2-iodomethyl-2-aryl epoxide proceeds with unusual retention of stereoconfiguration.
Halogenative functionalization of olefins is one of the most important transformations in organic synthesis, as it not only provides a versatile handle for further derivatization, but also delivers highly diastereoselective ring closure when the nucleophile and alkene are tethered together. ${ }^{1}$ Even though applications of halogenation reactions in total synthesis are well documented, ${ }^{2}$ catalytic enantioselective halogenation remains a significant challenge due to the rapid interexchange of the halonium complex between olefins, which leads to rapid racemization of the enantiopure halonium intermediate. ${ }^{3}$ Therefore, limited success has been achieved, despite enormous efforts being devoted to asymmetric halogenation reactions. ${ }^{4}$ Very recently, there has been impressive progress in this field after the landmark reports of Borhan, ${ }^{5 a}$ Tang, ${ }^{5 b}$ Fujioka, ${ }^{5 c}$ Jacobsen, ${ }^{5 d}$ and Yeung ${ }^{5 e}$ in 2010, taking advantage of organocatalysts to effect asymmetric halo-lactonization. ${ }^{5}$ Organocatalyzed enantioselective halocyclization of olefinic amines,

${ }^{a}$ Shaanxi Key Laboratory of Natural Products \& Chemical Biology, College of Science, Northwest A\&F University, 22 Xinong Road, Yangling 712100, China. E-mail: xiewqsioc@aliyun.com

${ }^{b}$ State Key Laboratory of Natural Medicines, Jiangsu Key Laboratory of Drug Discovery for Metabolic Diseases, Center of Drug Discovery, China Pharmaceutical University, 24 Tongjiaxiang, Nanjing 210009, China

'Hubei Collaborative Innovation Center for Rare Metal Chemistry, Hubei Normal University, China

${ }^{d}$ Shanghai Institute of Organic Chemistry, Chinese Academy of Sciences, China

${ }^{e}$ School of Science \& Laboratory for Microstructures, Instrumental Analysis and Research Center, Shanghai University, China

$\dagger$ Electronic supplementary information (ESI) available: Experimental procedures and characterization for all new compounds. CCDC 1023013 and 1028455. For ESI and crystallographic data in CIF or other electronic format see DOI: $10.1039 / \mathrm{c} 5 \mathrm{sc} 02485 \mathrm{~d}$

$\ddagger$ These authors contributed equally to this work. alcohols and other substrates subsequently emerged. ${ }^{6-9}$ However, asymmetric halocyclization reactions are currently limited to the formation of four- to six-membered rings. ${ }^{5-9}$ The generation of enantioenriched, more strained three-membered rings via catalytic asymmetric halocyclization remains elusive. In this regard, although 3-exo halo-cycloetherification of allyl alcohols has long been known, ${ }^{10}$ reactive halogenating agents or harsh reaction conditions are needed to effect the energetically disfavored 3-exo halocyclization, which impedes the development of an asymmetric version of this reaction.

With the advent and booming of organocatalysis, ${ }^{11 a-c}$ ionpairing of organocatalysts has emerged as a powerful strategy for designing new efficient organocatalysts. ${ }^{11 d}$ By cooperatively activating reactive partners, ion-pair catalysts have catalyzed enantioselective reactions that are otherwise difficult to achieve using other organocatalysts. In addition, the ion-pairing strategy also enables catalyst screening via combinational approaches, which greatly accelerates the catalyst screening process. Inspired by Toste's recent work $^{8 b-f}$ and our work on enantioselective halogenation reactions using chiral anionic phase transfer catalysts, ${ }^{12}$ we postulated that an ion-pair catalyst could facilitate the enantioselective halogenation reaction by cooperative and synergistic activation of both reactants (Fig. 1), which has been responsible for the success of previous catalysts. ${ }^{5-9}$ To this end, chiral phosphate was judiciously chosen as counter anion for its fine-tunable chiral pocket as well as its Brønsted basicity to allow interaction with the substrate. ${ }^{8}$ Furthermore, DABCO-derived quaternary ammonium could serve as an excellent candidate for the cation moiety, since its tertiary amine moiety could act as a Lewis base to stabilize the halonium complex, an approach which has been utilized for the 
a) bifunctional ion-pair organocatalyst designing:

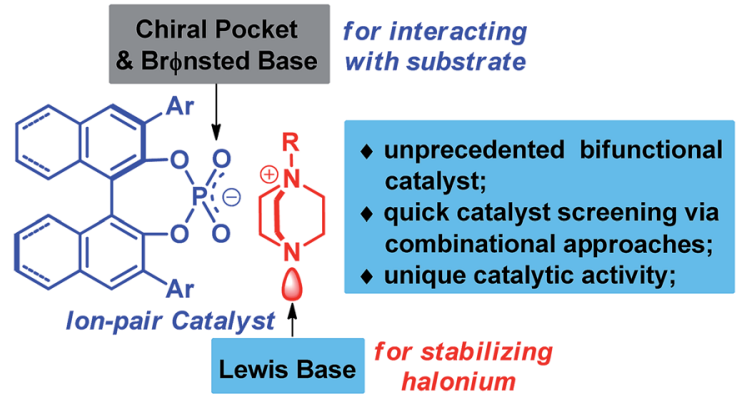

b) enantioselective 3-exo iodo-cycloetherification:

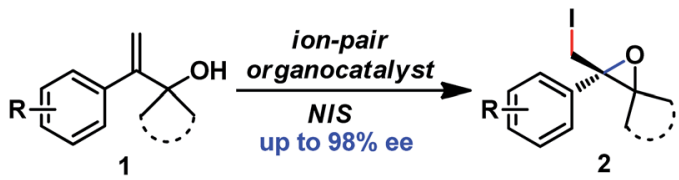

Fig. 1 Ion-pair organocatalyst design for enantioselective 3-exo iodocycloetherification of allyl alcohols. synthesis of well-known Selectfluor ${ }^{13}$ and other halogenating reagents. ${ }^{8 d, 9 c, 10 b}$

Herein, we would like to report the success of implementation of the ion-paring strategy, leading to the discovery of a novel ion-pair organocatalyst. This unprecedented organocatalyst enables the first enantioselective 3-exo iodo-cycloetherification of allyl alcohols using commercially available NIS as a halogen source. Additionally, this protocol provides direct access to enantiopure 2-iodomethyl epoxides, ${ }^{\mathbf{1 4}}$ which have previously been tedious to prepare from allyl alcohols, requiring an asymmetric Sharpless epoxidation/hydroxyl transformation procedure. $^{15}$

To validate our hypothesis, enantioselective 3-exo-iodocyclization of allyl alcohol 1a was explored using an ion-pair organocatalyst generated in situ by combining silver phosphate with DABCO-derived quaternary ammonium salt for convenience of catalyst screening (Table 1). Initially, various ammonium salts were evaluated using $8 H$-R-TRIP-OAg L1 as a chiral counteranion source. After extensive screening, A3 was determined to be a privileged scaffold, affording epoxide $2 \mathrm{a}$ with $77 \%$ ee in

Table 1 Optimization of reaction conditions for enantioselective 3-exo-iodocyclization of allyl alcohol $1 a^{a}$
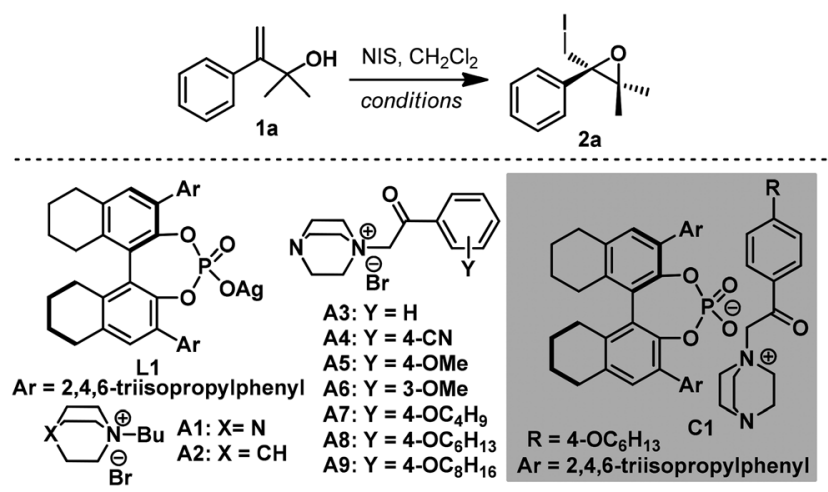

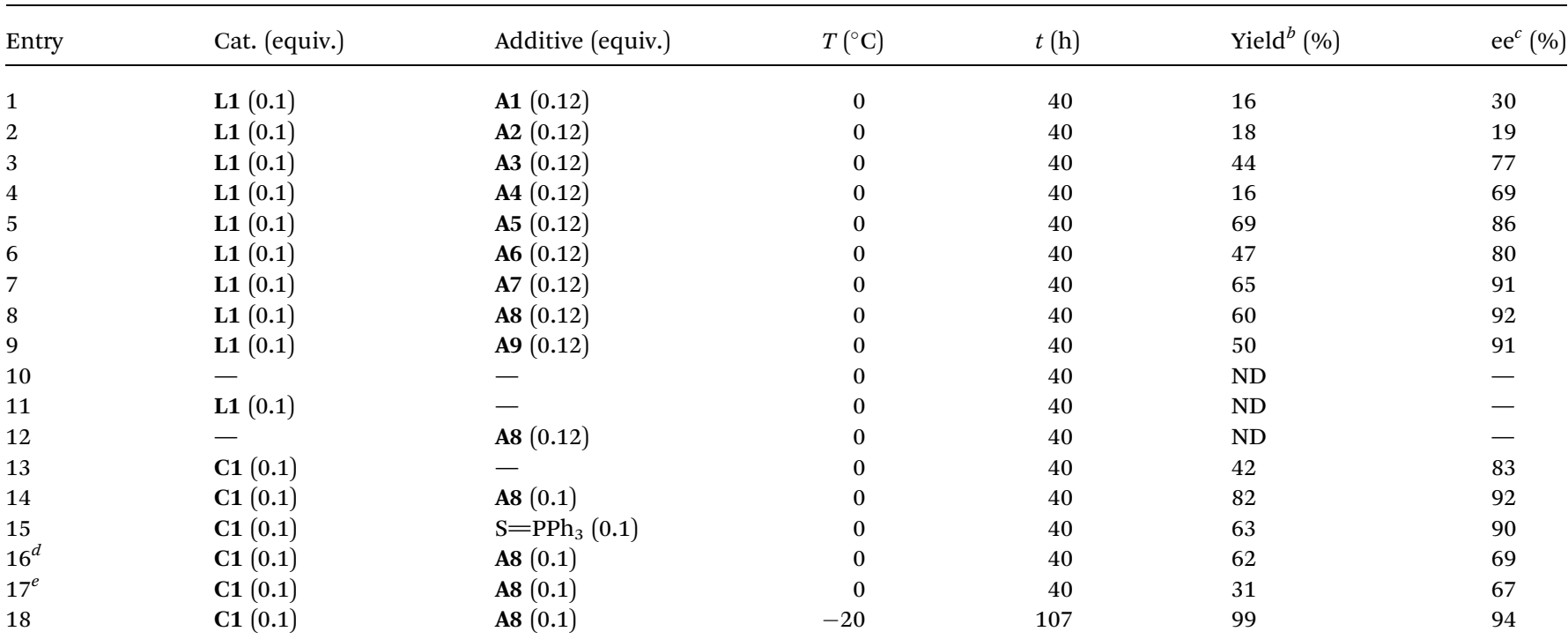

${ }^{a} \mathrm{CH}_{2} \mathrm{Cl}_{2}(1 \mathrm{~mL})$ was added to a mixture of silver salt $\mathbf{L 1}(0.01 \mathrm{mmol})$, ammonium salt $\mathbf{A}(0.012 \mathrm{mmol})$ and NIS $(0.12 \mathrm{mmol})$, and the reaction mixture was cooled to $0{ }^{\circ} \mathrm{C}$. Allyl alcohol $1 \mathrm{a}(0.1 \mathrm{mmol})$ in $0.5 \mathrm{~mL} \mathrm{CH}_{2} \mathrm{Cl}_{2}$ was then added dropwise, and the reaction was quenched at the indicated time.

${ }^{b}$ Isolated yield. ${ }^{c}$ Determined by HPLC using a Chiralpak AD column. ${ }^{d} \mathrm{CHCl}_{3}$ as solvent. ${ }^{e}$ EtOAc as solvent. ND $=$ not detected. 

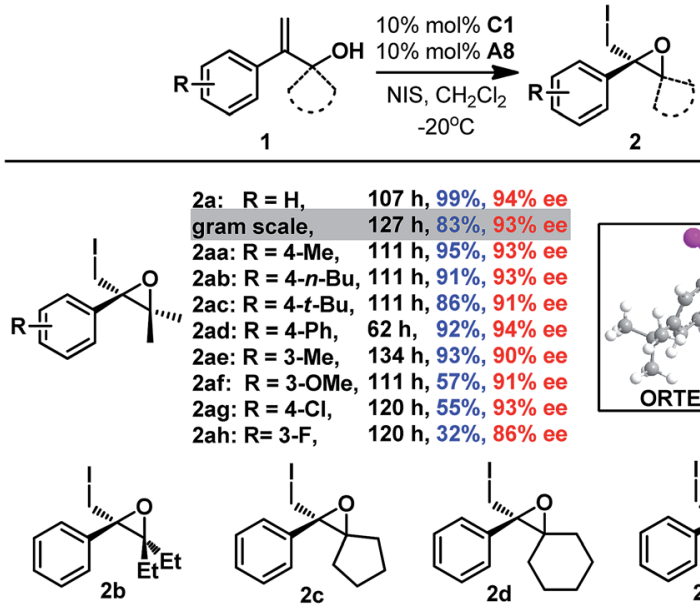

2a: $\quad \mathbf{R}=\mathbf{H}, \quad 107 \mathrm{~h}, 99 \%, 94 \%$ ee gram scale, $\quad 127 \mathrm{~h}, 83 \%, 93 \%$ ee 2aa: $\mathbf{R}=4-\mathrm{Me}, \quad 111 \mathrm{~h}, 95 \%, 93 \%$ ee 2ab: $\mathbf{R}=4-n-B u, 111$ h, 91\%, 93\% ee 2ac: $R=4-t-B u, 111$ h, $86 \%, 91 \%$ ee 2ad: $\mathbf{R}=4-\mathrm{Ph}, \quad 62 \mathrm{~h}, \quad 92 \%, 94 \%$ ee 2ae: $R=3-M e, \quad 134$ h, 93\%, 90\% ee 2af: $\mathbf{R}=3-\mathrm{OMe}, 111 \mathrm{~h}, \mathbf{5 7 \%}, 91 \%$ ee 2ag: $\mathbf{R}=\mathbf{4}-\mathbf{C l}, \quad \mathbf{1 2 0} \mathrm{h}, \mathbf{5 5} \%, \mathbf{9 3} \%$ ee 2ah: R= 3-F, $\quad 120$ h, 32\%, 86\% ee
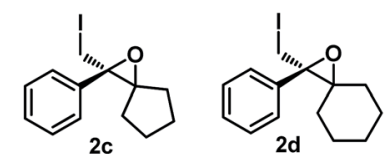

100 h, $60 \%, 95 \%$ ee 44 h, $99 \%, 97 \%$ ee gram scale

$2 d$ gram scale $48 \mathrm{~h}, 90 \%, 90 \%$
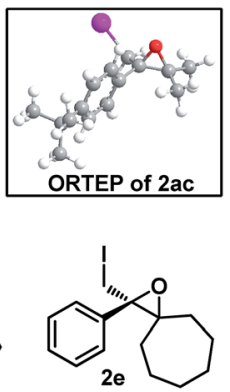

$91 \%, 98 \%$ 7 h, $92 \%, 98 \%$ ee 48 h, $90 \%, 90 \%$ ee $\mathbf{5 0}$ h, $89 \%, 98 \%$ ee

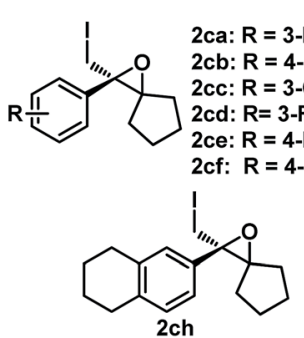

45 h, 76\%, 91\% ee
$2 \mathrm{~h}, 84 \%, 95 \%$ ee

\section{西}
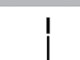
h, $81 \%, 96.5 \%$ ee h, $81 \%, 96.5 \%$ ee
h, $97 \%, 96.5 \%$ ee
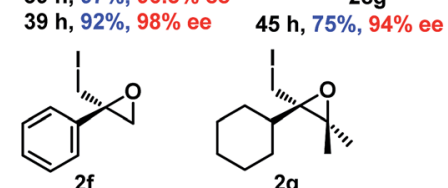

56 h, $41 \%, 63 \%$ ee

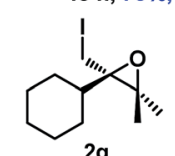

$74 \mathrm{~h}, 71 \%, 37 \%$ ee

Scheme 1 Substrate variation in the enantioselective 3-exo iodocycloetherification of allyl alcohols.

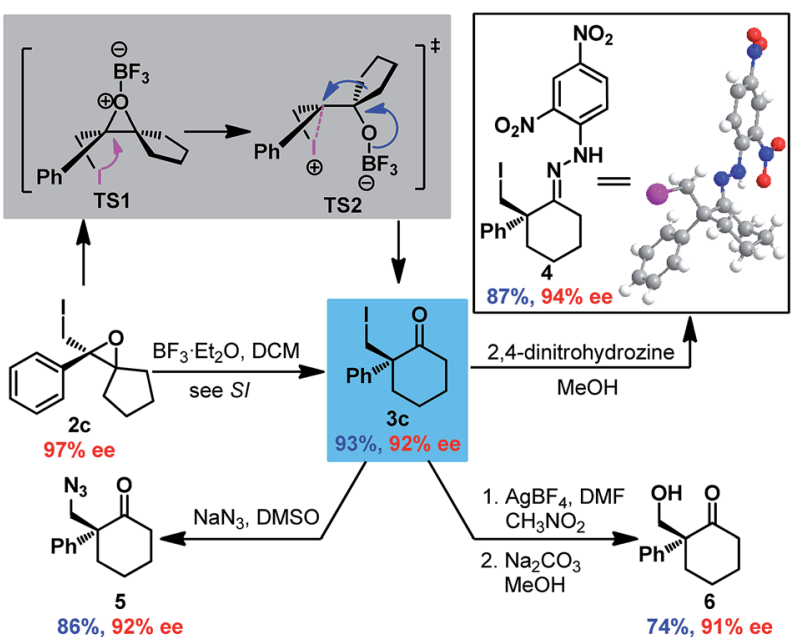

Scheme 2 Transformations of spiro-epoxide 2c.

moderate yield (entries 1-3 and ESI $\dagger$ ). In contrast, ammonium salt A2 derived from quinuclidine provided lower enantioselectivity, showing that the tertiary amine moiety of $\mathbf{A 1}$ played a pivotal role in the reaction (entries 1 and 2). Further structural modification of ammonium salt $\mathbf{A 3}$ revealed that $\mathbf{A 8}$ was the optimal cation fragment for the ion-pair organocatalyst, furnishing epoxide 2 a with $92 \%$ ee (entries 3-9). As for the anion fragment, $8 H$ - $R$-TRIP-OAg provided a better result than any other chiral silver phosphate evaluated (see ESI $\dagger$ ). Importantly, both cationic and anionic fragments were indispensable for the reaction, as indicated by control experiments (entries 10-12). It should be pointed out that other organocatalysts (e.g. chiral phosphoric acid and quinine-derived catalysts) were also surveyed under identical reaction conditions but gave no desired cyclization product, with the starting material being fully recovered (Table S2, ESI $\dagger$ ).

With the optimal anionic and cationic moiety of the catalyst identified, ion-pair organocatalyst $\mathbf{C 1}$ was synthesized directly from $8 H$ - $R$-TRIP and ammonium A8 (see ESI $\dagger$ ) and examined under otherwise identical reaction conditions. To our surprise, 2a was obtained with only moderate enantioselectivity ( $83 \%$ ee, entry 13). As a slight excess of $\mathbf{A 8}$ was used in the in situ procedure, we reasoned that $\mathbf{A 8}$ might be an effective promoter for this reaction. Indeed, comparable enantioselectivity (92\% ee, entry 14) was obtained by adding a catalytic amount of $\mathbf{A 8}$ to the reaction. It is postulated that $\mathbf{A 8}$ might act as a Lewis base to stabilize the iodonium intermediate ${ }^{8 \boldsymbol{d}}$ and facilitate the transfer of iodine from NIS to the DABCO moiety of the ion-pair organocatalyst, leading to an acceleration of the reaction rate and increased enantioselectivity. Employing $\mathrm{S}=\mathrm{PPh}_{3}$ (ref. 7c and e) as an additive also gave a comparable result, verifying the positive effect of a Lewis base as co-catalyst in this reaction (entry 15). With a suitable catalyst in hand, other reaction variations were subsequently evaluated. Other halogenating reagents such as NCS and NBS gave inferior results, leading to no reaction or a sharp drop in enantioselectivity (see $\mathrm{ESI}_{\dagger} \dagger$ ). $\mathrm{CH}_{2} \mathrm{Cl}_{2}$ was determined to be the optimal solvent (entries 16, 17 and ESI $\dagger$ ), and lowering the reaction temperature to $-20{ }^{\circ} \mathrm{C}$ was beneficial for the reaction (entry 18).

After establishing the optimal reaction conditions, the substrate scope of this reaction was examined (Scheme 1). Both electron-withdrawing groups (2aa-2af and 2ce-2cf) and electron-donating groups (2ag-2ah and $\mathbf{2 c a - 2 c h ) ~ o n ~ t h e ~ p h e n y l ~}$ moiety were tolerated, affording the corresponding epoxides with good to excellent enantioselectivities ( $87 \%$ to $99 \%$ ee). Gem-substituents were crucial for the reaction, as $2 \mathbf{f}$ lacking gem-substituents was obtained in only $41 \%$ yield and $63 \%$ ee. Epoxides with cyclic gem-substituents were obtained with higher enantioselectivities (2c-2ch and ESI $\dagger$ ) than those with acyclic gem-substituents (2a and 2b). A 2-alkyl substituted allyl alcohol was also smoothly converted to epoxide $2 \mathbf{g}$, albeit with low enantioselectivity ( $37 \%$ ee). Furthermore, gram syntheses of epoxides $2 \mathbf{a}$ and $\mathbf{2 c - 2 e}$ were also smoothly realized by using 5 mol\% $\mathbf{C 1}$ without affecting enantioselectivities, and the catalyst loading could even be reduced to $1 \mathrm{~mol} \%$ affording comparable results (Scheme 1 and $\mathrm{ESI}_{\dagger}^{\dagger}$ ). The absolute configuration of epoxide 2 was determined to be $R$ based on X-ray crystallographic analysis of epoxide $2 \mathbf{a c},{ }^{16}$ which was confirmed by vibrational circular dichroism (VCD) studies of epoxide 2c. ${ }^{17}$

Next, Wagner-Meerwein rearrangement ${ }^{18}$ of epoxide $2 \mathrm{c}$ was explored for the construction of 2-iodomethyl-2-aryl cyclohexanones with a chiral quaternary carbon center (Scheme 2). $\mathrm{BF}_{3}$ $\cdot \mathrm{Et}_{2} \mathrm{O}$ was determined to be the most efficient promoter (see ESI $\dagger$ ), delivering cyclohexanone $3 \mathbf{c}$ in good yield with partial 


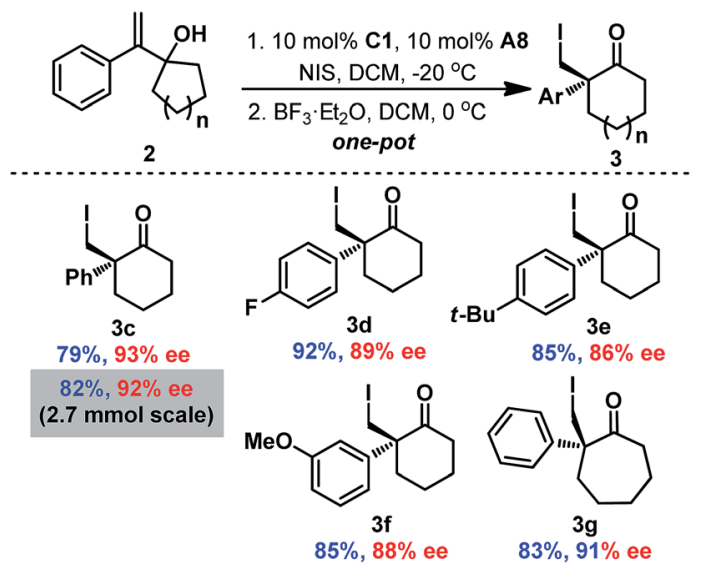

Scheme 3 One-pot asymmetric 3-exo iodo-cycloetherification/ Wagner-Meerwein rearrangement reaction.

loss of enantioselectivity (93\% ee vs. $97 \%$ ee for epoxide 2 c). Surprisingly, the absolute configuration of $3 \mathbf{c}$ was established to be $S$ by X-ray crystallographic analysis of hydrazone 4 derived from $3 \mathbf{c},{ }^{16}$ which indicated retention of stereoconfiguration in the Wagner-Meerwein rearrangement. This could be ascribed to the opening of the epoxide by the adjacent iodine to generate iodonium TS2, which then rearranged to ketone $3 \mathbf{c}$ with double inversion of configuration. Furthermore, derivatizations of $3 \mathbf{c}$ were also performed to demonstrate its synthetic utility. Substitution of the iodide with $\mathrm{NaN}_{3}$ provided azide ketone $\mathbf{5}$ smoothly, and the iodide could also be converted to an alcohol via formyloxylation/hydrolysis ${ }^{19}$ to give hydroxyl ketone 6 in satisfactory yield. It is noteworthy that no erosion of enantiopurity was detected in all these reactions.

To simplify the operation, one-pot asymmetric 3-exo iodocycloetherification/Wagner-Meerwein rearrangement was also developed (Scheme 3). Fortunately, when the iodo-cycloetherification reaction was completed, addition of $\mathrm{BF}_{3} \cdot \mathrm{OEt}_{2}$ to the reaction mixture smoothly provided the desired cyclohexanone 3c without reducing enantioselectivity, even on a $2.7 \mathrm{mmol}$ scale (92\% ee). Different substituents on the phenyl group were found to be compatible with the one-pot process, affording the corresponding cyclohexanones $\mathbf{3 c - 3 f}$ in satisfactory enantiopurities. Furthermore, seven-membered cycloketone $\mathbf{3 g}$ could also be obtained via this one-pot cascade reaction with $91 \%$ ee (comparable with that of the corresponding epoxide 2d), providing a complementary route to previous protocols involving enantioselective haloniuminduced semi-Pinacol rearrangement for the enantioselective construction of halogenated cycloheptanones. ${ }^{9 a-e}$

\section{Conclusions}

In conclusion, a novel ion-pair organocatalyst comprised of chiral phosphate and DABCO-derived quaternary ammonium was designed, which enabled the first asymmetric 3-exo iodocycloetherification of allyl alcohols using NIS as a halogenating reagent. By employing this novel catalyst, a variety of enantiopure 2-iodomethyl-2-aryl epoxides were successively prepared with good to excellent enantioselectivities, even on a gram scale. Subsequently, one-pot asymmetric 3-exo iodo-cycloetherification/Wagner-Meerwein rearrangement of 2-aryl-2propen-3-ol was explored, which provided direct access to chiral 2-iodomethyl-2-aryl cycloalkanones with good enantioselectivities. Unusual retention of configuration owing to the assistance of the adjacent iodide was also observed in the Wagner-Meerwein rearrangement.

\section{Acknowledgements}

We are grateful for financial support from the National Natural Science Foundation of China (grant No. 21372239, 21202187) and the Scientific Research Foundation of Northwest A\&F University (grant No. Z111021501).

\section{Notes and references}

1 (a) F. A. Carey and R. J. Sundberg, Advanced Organic Chemistry, Part B, 5th edn, Plenum, New York, 2007, p. 298; (b) R. C. Larock, Comprehensive Organic Transformations, 2nd edn, Wiley-VCH, New York, 1999, p. 638.

2 For selected recent examples, see: (a) D. K. Bedke, G. M. Shibuya, A. Pereira, W. H. Gerwick, T. H. Haines and C. D. Vanderwal, J. Am. Chem. Soc., 2009, 131, 7570; (b) S. A. Snyder, Z.-Y. Tang and R. J. Gupta, J. Am. Chem. Soc., 2009, 131, 5744; (c) C. Nilewski, R. W. Geisser and E. M. Carreira, Nature, 2009, 457, 573.

3 (a) R. S. Brown, R. W. Nagorski, A. J. Bennet, R. E. D. Mcclung, G. H. M. Aarts, M. Klobukowski, R. McDonald and B. D. Santarsiero, J. Am. Chem. Soc., 1994, 116, 2448; (b) A. A. Neverov and R. S. Brown, J. Org. Chem., 1996, 61, 962; (c) R. S. Brown, Acc. Chem. Res., 1997, 30, 131; (d) S. E. Denmark, M. T. Burk and A. J. Hoover, J. Am. Chem. Soc., 2010, 132, 1232.

4 For selected examples, see: (a) I. Sakurada, S. Yamasaki, R. Gottlich, T. Iida, M. Kanai and M. Shibasaki, J. Am. Chem. Soc., 2000, 122, 1245; (b) A. K. El-Qisairi, H. A. Qaseer, G. Katsigras, P. Lorenzi, U. Trivedi, T. S. Tracz, A. Hartman, J. A. Miller and P. M. Henry, Org. Lett., 2003, 5, 439; (c) S. H. Kang, S. B. Lee and C. M. Park, J. Am. Chem. Soc., 2003, 125, 15748; (d) A. Sakakura, A. Ukai and K. Ishihara, Nature, 2007, 445, 900.

5 For enantioselective halo-lactonization reactions, see: $(a)$ D. C. Whitehead, R. Yousefi, A. Jaganathan and B. Borhan, J. Am. Chem. Soc., 2010, 132, 3298; (b) W. Zhang, S. Zheng, N. Liu, J. B. Werness, I. A. Guzei and W. Tang, J. Am. Chem. Soc., 2010, 132, 3664; (c) K. Murai, T. Matsushita, A. Nakamura, S. Fukushima, M. Shimura and H. Fujioka, Angew. Chem., Int. Ed., 2010, 49, 9174; (d) G. E. Veitch and E. N. Jacobsen, Angew. Chem., Int. Ed., 2010, 49, 7332; (e) L. Zhou, C. K. Tan, X. Jiang, F. Chen and Y.-Y. Yeung, J. Am. Chem. Soc., 2010, 132, 15474; $(f)$ M. C. Dobish and J. N. Johnston, J. Am. Chem. Soc., 2012, 134, 6068; $(g)$ X. Jiang, C. K. Tan, L. Zhou and Y.-Y. Yueng, Angew. Chem., Int. Ed., 2012, 51, 7771; (h) D. H. Paull, C. Fang, J. R. Donald, A. D. Pansick and S. F. Martin, J. Am. Chem. 
Soc., 2012, 134, 11128; (i) K. Ikeuchi, S. Ido, S. Yoshimura, T. Asakawa, M. Inai, Y. Hamashima and T. Kan, Org. Lett., 2012, 14, 6016; (j) M. Wilking, C. Mück-Lichtenfeld, C. G. Daniliuc and U. Hennecke, J. Am. Chem. Soc., 2013, 135, 8133.

6 For reviews, see: (a) A. Castellanos and S. P. Fletcher, Chem.Eur. J., 2011, 17, 5766; (b) C. K. Tan, L. Zhou and Y.-Y. Yeung, Synlett, 2011, 1335; (c) U. Hennecke, Chem.-Asian J., 2012, 7, 456; (d) S. E. Denmark, W. E. Kuester and M. T. Burk, Angew. Chem., Int. Ed., 2012, 51, 10938; (e) C. K. Tan and Y.-Y. Yeung, Chem. Commun., 2013, 49, 7985; $(f)$ K. Brak and E. N. Jacobsen, Angew. Chem., Int. Ed., 2013, 52, 534; $(g)$ J. Chen and L. Zhou, Synthesis, 2014, 46, 586.

7 For halo-cycloetherification reactions, see: (a) U. Hennecke, C. H. Müller and R. Frölich, Org. Lett., 2011, 13, 860; (b) D. Huang, H. Wang, F. Xue, H. Guan, L. Li, X. Peng and Y. Shi, Org. Lett., 2011, 13, 6350; (c) S. E. Denmark and M. T. Burk, Org. Lett., 2012, 14, 256; (d) X. H. Zeng, C. X. Miao, S. F. Wang, C. G. Xia and W. Sun, Chem. Commun., 2013, 49, 2418; (e) S. E. Denmark and M. T. Burk, Chirality, 2014, 26, 344; (f) D. W. Tay, G. Y. C. Leung and Y.-Y. Yeung, Angew. Chem., Int. Ed., 2014， 53，5161; $(g)$ Z. Ke, C. K. Tan, F. Chen and Y.-Y. Yeung, J. Am. Chem. Soc., 2014, 136, 5627.

8 For chiral phosphate directed reactions, see: $(a)$ R. J. Phipps, G. L. Hamilton and F. D. Toste, Nat. Chem., 2012, 4, 603; (b) M. Mahlau and B. List, Angew. Chem., Int. Ed., 2013, 52, 518. For selected halogenation reactions using chiral phosphoric acid as a phase transfer catalyst, see: (c) V. Rauniyar, A. D. Lackner, G. L. Hamilton and F. D. Toste, Science, 2011, 334, 1681; (d) Y.-M. Wang, J. Wu, C. Hoong, V. Rauniyar and F. D. Toste, J. Am. Chem. Soc., 2012, 134, 12928; (e) T. Honjo, R. J. Phipps, V. Rauniyar and F. D. Toste, Angew. Chem., Int. Ed., 2012, 51, 9684; $(f)$ H. P. Shunatona, N. Früh, Y.-M. Wang, V. Rauniyar and F. D. Toste, Angew. Chem., Int. Ed., 2013, 52, 7724.

9 For halonium induced semi-pinacol rearrangements, see: $(a)$ Z.-M. Chen, Q.-W. Zhang, Z.-H. Chen, H. Li, Y.-Q. Tu, F.-M. Zhang and J.-M. Tian, J. Am. Chem. Soc., 2011, 133, 8818; (b) C. H. Muller, M. Wilking, A. Ruhlmann, B. Wibbeling and U. Hennecke, Synlett, 2011, 2043; (c) F. Romanov-Michailidis, L. Guénée and A. Alexakis, Org. Lett., 2013, 15, 5890; (d) F. Romanov-Michailidis, L. Guénée and A. Alexakis, Angew. Chem., Int. Ed., 2013, 52, 9266; (e) Q. Yin and S.-L. You, Org. Lett., 2014, 16, 1810. For selected other reactions, see: (f) K. C. Nicolaou, N. L. Simmons, Y. Ying, P. M. Heretsch and J. S. Chen, J. Am. Chem. Soc.,
2011, 133, 8134; $(g)$ F. Chen, C. K. Tan and Y.-Y. Yeung, $J$. Am. Chem. Soc., 2013, 135, 1232; (h) K. Mori, Y. Ichikawa, M. Kobayashi, Y. Shibata, M. Yamanaka and T. Akiyama, J. Am. Chem. Soc., 2013, 135, 3964; (i) Y. Zhao, X. Jiang and Y.-Y. Yeung, Angew. Chem., Int. Ed., 2013, 52, 8597; (j) D. Huang, X. Liu, L. Li, Y. Cai, W. Liu and Y. Shi, J. Am. Chem. Soc., 2013, 135, 8101; (k) C. S. Brindle, C. S. Yeung and E. N. Jacobsen, Chem. Sci., 2013, 4, 2100.

10 For selected examples, see: (a) F. Straus and R. Kuhnel, Chem. Ber., 1933, 66, 1834; (b) R. D. Evans, J. W. Magee and J. H. Schauble, Synthesis, 1988, 862; (c) P. Galatsis and S. D. Millan, Tetrahedron Lett., 1991, 32, 7493; (d) I. Rawal, Tetrahedron Lett., 1992, 33, 4687.

11 For selected reviews, see: (a) New Frontiers in Asymmetric Catalysis, ed. K. Mikami and M. Lautens, WileyInterscience, Hoboken, NJ, 2007, vol. 3; (b) Enantioselective Organocatalysis, ed. P. I. Dalko, Wiley-VCH, Weinheim, 2007; (c) K. L. Jensen, G. Dickmeiss, H. Jiang, L. Albrecht and K. A. Jørgensen, Acc. Chem. Res., 2012, 45, 248; (d) J.-F. Brière, S. Oudeyer, V. Dallab and V. Levacher, Chem. Soc. Rev., 2012, 41, 1696.

12 (a) W. Xie, G. Jiang, H. Liu, J. Hu, X. Pan, H. Zhang, X. Wan, Y. Lai and D. Ma, Angew. Chem., Int. Ed., 2013, 52, 12924; (b) H. Liu, G. Jiang, X. Pan, X. Wan, Y. Lai, D. Ma and W. Xie, Org. Lett., 2014, 16, 1908.

13 G. S. Lal, J. Org. Chem., 1993, 58, 2791-2796.

14 For a recent review on organocatalyzed epoxidation, see: R. L. Davis, J. Stiller, T. Naicker, H. Jiang and K. A. Jögensen, Angew. Chem., Int. Ed., 2014, 53, 7406.

15 For selected examples, see: (a) T. Ichige, Y. Okano, N. Kanoh and M. Nakata, J. Am. Chem. Soc., 2007, 129, 9862; (b) A. R. van Dyke and T. F. Jamison, Angew. Chem., Int. Ed., 2009, 48, 4430; (c) Q. Yang, J. T. Njardarson, C. Draghici and F. Li, Angew. Chem., Int. Ed., 2013, 52, 8648.

16 CCDC 1023013 and 1028455 contain the supplementary crystallographic data for compounds 2 ac and 4 , respectively. For a rationale of the observed stereoselectivity, see the ESI. $\dagger$

17 See ESI $\dagger$ for detailed experimental data.

18 For selected examples, see: (a) Y. Kita, K. Higuchi, Y. Yoshida, K. Iio, S. Kitagaki, K. Ueda, S. Akai and H. Fujioka, J. Am. Chem. Soc., 2001, 123, 3214; (b) Y. M. Shen, B. Wang and Y. Shi, Angew. Chem., Int. Ed., 2006, 45, 1429.

19 A. Abad, C. Agulló, A. C. Cuñat and I. Navarro, Synthesis, 2005, 3355. 